\title{
Congo-Crimean haemorrhagic fever in Dubai: histopathological studies
}

\author{
A BASKERVILle,* AGO SATTI,† FA MURPHY, $\ddagger$ DIH SIMPSON*
}

From the *PHLS Centre for Applied Microbiology and Research, Porton Down, Salisbury, Wiltshire SP4 OJG, England, the $\dagger E l$ Rashid Hospital, Dubai, United Arab Emirates, and the $\ddagger$ College of Veterinary Medicine and Biomedical Sciences, Colorado State University, Fort Collins, Colorado 80523, USA

SUMMARY Necropsies were carried out on two patients who died of Congo-Crimean haemorrhagic fever (C-CHF) in Dubai. The diagnosis was confirmed by isolation of C-CHF virus from the liver. Histopathological changes included extensive cellular necrosis and haemorrhage in the liver, necrosis and lymphoid depletion in the spleen, congestion and oedema formation in the lungs, and haemorrhage in a number of other organs.

Although Crimean haemorrhagic fever was first recognised in Russia in 1944, and an identical virus (Congo) was isolated in Africa in 1956, the disease has been identified only comparatively recently in the Middle East. Three notable hospital-associated outbreaks have taken place which have led to deaths among hospital staff. The first of these episodes occurred in Pakistan, ${ }^{1}$ when a patient underwent an exploratory operation for haematemesis; four members of the operating team and an attendant physician became infected and two of them died. A limited outbreak occurred in Baghdad in 1979, in which the index case was a patient with a threatened abortion and severe bleeding. Two of the hospital staff attending the patient became ill and died. ${ }^{2}$

The third outbreak was in Dubai. ${ }^{3}$ Here the index case presented in the casualty department with fever, dyspnoea, bleeding gums, oral congestion and ulceration. Shortly after admission he had a cardiorespiratory arrest and a massive melaena of fresh blood. Vigorous, but unsuccessful, attempts were made to resuscitate the patient. Five to 10 days later, five members of the hospital staff who had attended this patient developed similar illnesses beginning with non-specific symptoms of fever, vomiting and diarrhoea later accompanied by severe bleeding from the nose, gums, gastrointestinal and urinary tracts. The most characteristic finding was the appearance of large ecchymoses on the forearms accompanied by marked thrombocytopenia. Two of the hospital staff died and necropsies were carried out on both patients. This paper presents the histopathological findings.

Accepted for publication 12 January 1981

\section{Material and methods}

Necropsies were carried out using full protective clothing, including respirators, caps, gowns, rubber aprons, gloves, and rubber boots. Tissues for histopathology were fixed in $10 \%$ buffered neutral formalin, processed by standard procedures and embedded in paraffin wax. Sections cut at $5 \mu \mathrm{m}$ were stained by haematoxylin and eosin, and selected sections were also stained by the following methods: periodic acid-Schiff (PAS), phosphotungstic acidhaematoxylin (PTAH), Machiavello, Gordon and Sweet, Verhoeff-van Gieson and Perl's Prussian Blue.

\section{Results}

Gross necropsy findings were those of generalised haemorrhage. Large purpuric areas were present in the antecubital fossae and on the buttocks and legs. One of the patients had massive haemorrhages in the stomach and small intestine, and the other had haemorrhages in the adrenals. This latter patient also had bilateral pleural effusions and ascites.

\section{VIRUS ISOLATION}

Congo-Crimean haemorrhagic fever virus was isolated from post-mortem liver specimens from one of the fatal cases. The virus was identified by immunofluorescence and by complement-fixation tests. $^{3}$ Rising antibody titres to $\mathrm{C}$-CHF virus were also demonstrated in three of the surviving patients.

\section{HISTOPATHOLOGY}

Liver The liver of both patients exhibited widespread hepatocellular necrosis, which was associated 

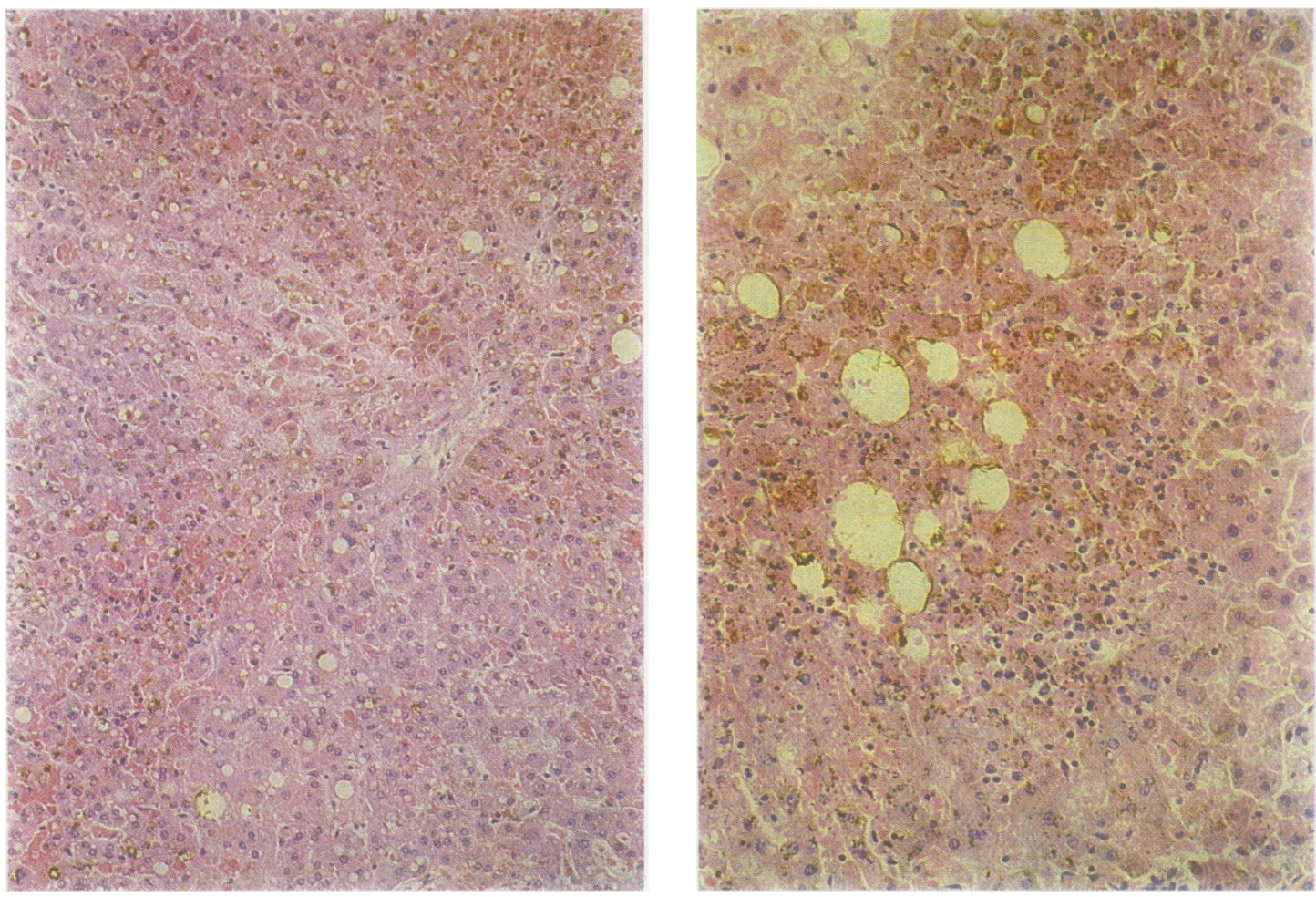

Fig. 1

Fig. 2
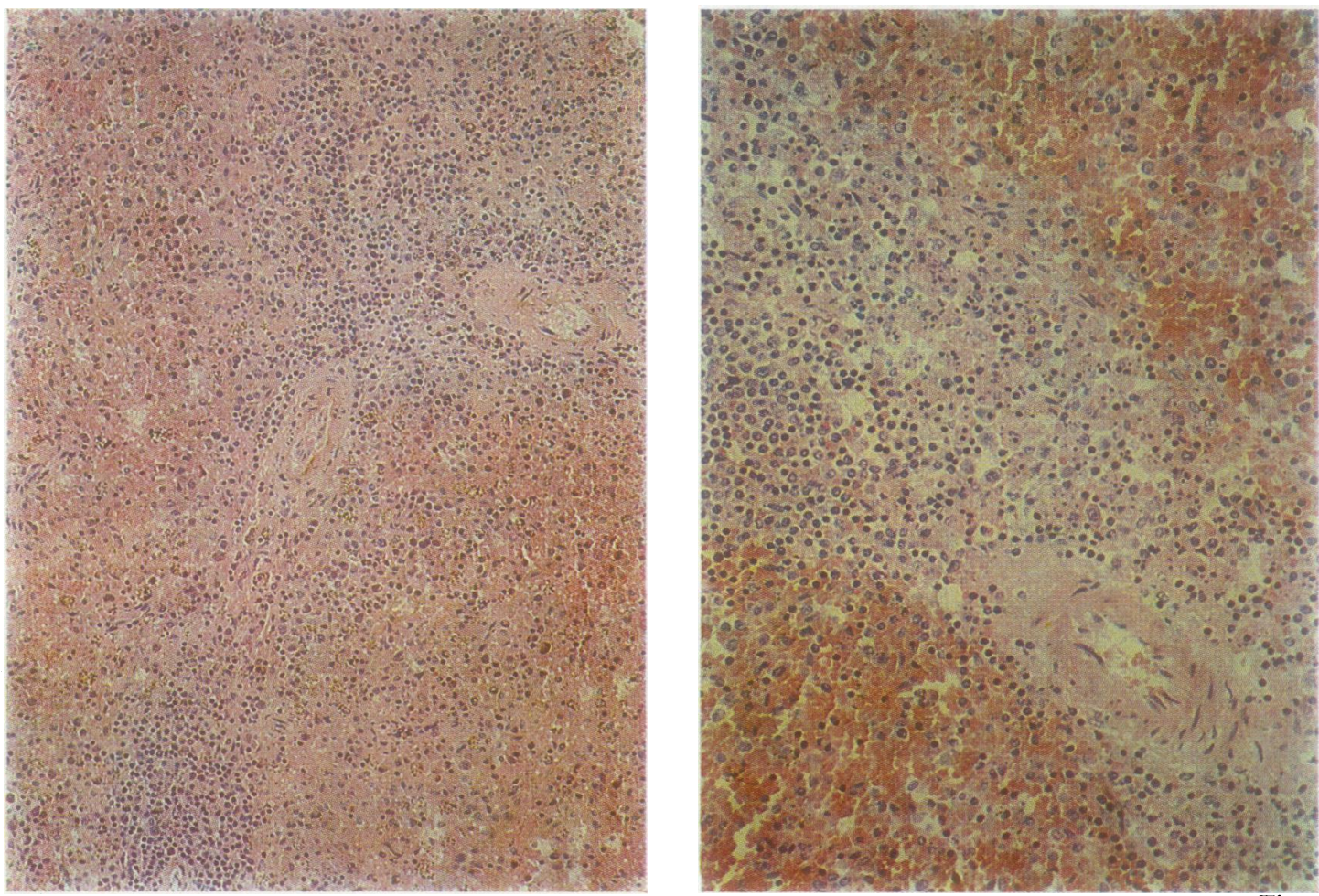

Fig. 3

Fig. 4

Fig. 1 Liver from $\mathrm{C}$-CHF patient showing eosinophilic necrosis of hepatocytes, associated with small haemorrhages. There is extensive deposition of bile and iron pigments. Haematoxylin and eosin $\times 70$

Fig. 2 Liver. Margin of an area of haemorrhagic necrosis with an intense Kupffer cell response. Haematoxylin and eosin $\times 120$

Fig. 3 Spleen of $C$-CHF patient. There is necrosis of white and red pulp and depletion of lymphoid elements.

Germinal centres are absent. Haematoxylin and eosin $\times 80$

Fig. 4 Spleen, showing necrosis of lymphocytes in the periarteriolar sheath. Haematoxylin and eosin $\times 100$ 
with a variable degree of haemorrhage in many sites (Fig. 1). Necrosis was predominantly mid-zonal, but other regions of the lobule were affected and in some areas the entire lobule was involved. The necrosis was eosinophilic, with the formation of large, amorphous, anucleate masses, similar in appearance to Councilman bodies (Fig. 2). The application of Machiavello's stain to the liver sections did not reveal inclusion bodies, the reticulin pattern was normal and sections stained by Verhoeffvan Gieson were unremarkable. Much of the remaining liver tissue showed moderate fatty change. There was no cellular inflammatory response in the lobules, and only a minimal periportal infiltration by lymphocytes and histiocytes. Kupffer cells in areas of haemorrhage were reactive and in sections stained by Perl's Prussian Blue were seen to contain iron pigment. Numerous bile thrombi were present throughout the specimens.

Spleen The predominant features were extreme dilatation of sinusoids, necrosis of red and white pulp, necrosis of marginal lymphocytes and generalised lymphoid depletion (Figs. 3 and 4). Karyorrhectic debris was present in the marginal zones in many areas. Reticulum cells were scarce in both cases. Remaining white pulp also lacked germinal centres and there were large deposits of haemosiderin.

Lungs The lungs of both patients were intensely congested and there was widespread intra-alveolar oedema formation, with fibrin deposition and small intra-alveolar haemorrhages. Airway epithelium had been completely lost through post-mortem autolysis, but there was no evidence of inflammatory infiltration in any site, nor were intravascular thrombi detected in sections stained by PTAH.

Kidneys There was considerable autolysis. Numerous haemorrhages were present, principally between tubules in the outer cortex, but some had occurred from glomerular tufts and into the interstitial tissue between collecting ducts in the medulla. There was no cellular inflammatory response. PAS-positive casts were seen in a number of tubules and collecting ducts.

Intestine The epithelium had undergone extensive autolysis in both patients, but haemorrhages were seen in the deeper parts of the mucosa and submucosa.

Adrenals The adrenal glands contained haemorrhages in all layers of the cortex and medulla, though those in one patient were much more extensive. There were no other changes.

Myocardium Apart from small haemorrhages between fibres in some areas there were no lesions.

\section{Discussion}

The clinical aspects of the illness presented by the patients in this outbreak of C-CHF have been described in detail elsewhere. ${ }^{3}$ The illnesses had a biphasic course. In each case non-specific symptoms were seen in the early stages, followed on about the sixth day of illness by haemorrhage from the nose, mouth, and gastrointestinal tract and occasionally also from the kidneys. Large ecchymotic areas appeared on the forearms and legs. The haematological changes were fairly uniform, with marked thrombocytopenia and leukopenia. Despite even severe bleeding, haemoglobin concentrations remained fairly high, probably due to haemoconcentration. The bone marrow was hypercellular, with increased activity of the erythroid and myeloid series, but no evidence of disseminated intravascular coagulation was recognised.

In the early stages of illness diagnosis is difficult because of the non-specific presentation. Even when frank haemorrhages appear it may be difficult to distinguish Congo-Crimean haemorrhagic fever infections from those of other viral haemorrhagic fevers such as Lassa, Marburg, and Ebola. However, the appearance of large ecchymotic areas on the arms is characteristic of Congo-Crimean infections and the limited geographical distribution of these diseases may often assist diagnosis.

The histopathological changes in the liver and spleen, hepatocellular necrosis and lymphoid depletion and necrosis, were similar to those seen in a number of haemorrhagic fevers caused by different agents including Lassa fever, Marburg, and Ebola viruses. $^{4-6}$ In the liver the eosinophilic necrosis, the formation of anucleate Councilman-like bodies and the absence of a cellular inflammatory response were similar to the changes of both Yellow fever and Lassa fever. The areas with haemorrhage particularly mimicked the appearance of Yellow fever. Sections stained by Machiavello's method did not demonstrate viral inclusion bodies. However, this stain is generally considered to be unreliable in necropsy material.

Although widespread haemorrhage was a feature of C-CHF infection in these patients and there was thrombocytopenia, there was no histological evidence in the lungs, kidneys or other organs of disseminated intravascular coagulation (DIC). DIC does occur in some haemorrhagic fevers ${ }^{7}$ and is thought to play a significant role in the pathogenesis and clinical course of the diseases in some patients. Evidence of its occurrence in experimental Ebola haemorrhagic fever infection in monkeys has also been presented. ${ }^{8}$

The necrosis of lymphoid tissue in the spleen and the absence of germinal centres in the splenic white pulp are particularly interesting. From the standpoint of differential diagnosis this lesion would be extremely difficult to distinguish from the changes in 
lymphoid tissue in Lassa, Marburg, Ebola, and Yellow fever. It is possible that the inhibition of lymphoid tissue is because the virus of C-CHF has a particular affinity for lymphoid cells and multiplies in them. Such infection of lymphoid cells has been shown to occur in Dengue. Damage to the lymphoid system and depletion of its cells may also account, at least in part, for the absence of inflammatory cell infiltrates in the liver and other organs.

\section{References}

${ }^{1}$ World Health Organization. Viral haemorrhagic fever, Pakistan. Weekly Epidemiology Record 1976;51, No 33: 261-2.

${ }^{2}$ World Health Organization. Viral haemorrhagic fever in Iraq confirmed as Congo virus. Weekly. Epidemiology Record 1979;54, No 46:359.

${ }^{3}$ Suleiman Mustafa Nur El Huda, Muscat-Baron JN,
Harries JR, et al. Congo/Crimean haemorrhagic fever in Dubai: an outbreak at the Rashid Hospital. Lancet 1980; ii:939-41.

' Edington GM, White HA. The pathology of Lassa fever. Trans $R$ Soc Trop Med Hyg 1972;66:381.

" Gedigk P. Bechtelsheimer H, Korb G. Die pathologische Anatomie der "Marburg-virus" Krankheit. Dtsch Med Wochenschr 1969;14:68-77.

${ }^{6}$ World Health Organization. Ebola haemorrhagic fever in Sudan, 1976. Bull WHO 1978;56, No 2:247-70.

${ }^{7}$ Mckay DC, Margaretten W. Disseminated intravascular coagulation in virus diseases. Arch Intern Med 1967;120 129-52.

${ }^{8}$ Baskerville A, Bowen ETW, Platt GS, McArdell BL. Simpson DIH. The pathology of experimental Ebola virus infection in monkeys. $J$ Pathol 1978;125:131-8.

Requests for reprints to: Dr A Baskerville, PHLS Centre for Applied Microbiology and Research, Pathogenic Microbes Research Laboratory, Porton Down, Salisbury, Wiltshire SP4 0JG, England. 